ISSN 0103-5150

Fisioter. Mov., Curitiba, v. 25, n. 1, p. 47-54, jan./mar. 2012 Licenciado sob uma Licença Creative Commons

\title{
Aderência dos cursos de Fisioterapia da região Norte às Diretrizes Curriculares Nacionais
}

\author{
Adherence of the physiotherapy courses in the northern region based \\ on the National Curriculum Guidelines
}

\section{Renato da Costa Teixeira}

Fisioterapeuta, professor assistente IV, Universidade do Estado do Pará (UEPA), Belém, PA - Brasil, e-mail: teixeirarenato@globo.com

\section{Resumo}

Introdução: Após a aprovação das Diretrizes Curriculares Nacionais para os cursos de Fisioterapia (DCN/ Fisio), houve um expressivo aumento no número de cursos de graduação em Fisioterapia. A região Norte apresentou a maior taxa de crescimento desses cursos, entre 1999 e 2004, existindo atualmente 27 cursos nessa região. A DCN/FISIO trouxe uma mudança no paradigma de formação desse profissional, passando-se a buscar um perfil de egresso generalista, humanista, crítico e reflexivo. Materiais e métodos: Com a finalidade de analisarmos a aderência dos cursos de fisioterapia da região Norte às DCN/Fisio, realizamos uma análise crítica dos projetos pedagógicos (PPC) de dez cursos de Fisioterapia da região, com base na mesma metodologia utilizada pelo Instituto Nacional de Estudos e Pesquisas Educacionais Anísio Teixeira (Inep) em 2006, em estudo semelhante com os cursos de Enfermagem, Medicina e Odontologia. Resultados: A aderência média dos PPC às DCN/Fisio foi de 74\%. Encontramos como pontos fortes: a construção coletiva dos projetos pedagógicos, matriz curricular interdisciplinar ou a utilização de metodologias que propiciam essa prática, a integração entre teoria e prática, a presença de mecanismos de controle do Trabalho de Conclusão de Curso, maior abrangência das áreas de atuação profissional durante o estágio supervisionado, o incentivo à produção científica, o equilíbrio entre as cargas horárias dos conteúdos essenciais e a presença de regulamento das atividades complementares. Por sua vez, encontramos como fragilidades: o projeto pedagógico 
construído por grupos restritos, a transcrição literal de elementos das DCN/Fisio no PPC, metodologias de ensino não condizentes com a perspectiva de formação das DCN/Fisio.

Palavras-chave: Fisioterapia. Aderência. Projeto pedagógico. Diretriz Curricular Nacional

\section{Abstract}

Introduction: After approval of the National Curriculum Guide for courses in Physiotherapy (DCN/Fisio), there was a significant increase in the number of undergraduate courses in Physiotherapy. The Northern region had the highest growth rate of these courses between 1999 and 2004, there are currently 27 courses in this region. The DCN/Fisio brought a paradigm shift in this professional education, moving from one profile to seek egress general, humanist, critical and reflective. Materials and methods: In order to analyze the adherence of Physiotherapy courses in the North to the DCN/Fisio, conducted a review of the pedagogical projects (PPC) of ten courses of Physiotherapy in the region, based on the same methodology used by the Anísio Teixeira National Institute of Studies and Educational Research (Inep) in 2006, in a similar study with courses of Nursing, Medicine and Dentistry. Results: The mean adhesion of PPC to the DCN/Fisio was 74\%. We find as best points: the collective construction of pedagogical projects, interdisciplinary curriculum or the use of methodologies that enable this practice, the integration between theory and practice, the presence of mechanism to control end of course monographs, the more breadth professional actuation areas during period training, the incentive for scientific production and the balance between the teaching schedule of essential contents and the presence of complementary activities regulation. On the other hand, we point out as fragilities: the indication that the pedagogical project was constructed in restricted groups, the literal transcription of elements of the $D C N / F i s i o$ in the pedagogical project, the use of teaching methodologies that do not match the educational perspective of the DCN/Fisio.

Keywords: Physiotherapy. Adherence. Pedagogical project of the course. National Curriculum Guidelines.

\section{Introdução}

Este artigo objetiva analisar a aderência dos projetos pedagógicos dos cursos de graduação em Fisioterapia da região Norte às Diretrizes Curriculares Nacionais (DCN/Fisio). 0 projeto pedagógico constitui o elemento balizador das práticas pedagógicas, devendo estar em consonância com as DCN. Projeto pedagógico não é uma expressão nova; ela passou a ser usada mais intensamente a partir da aprovação da Lei de Diretrizes e Bases da Educação Nacional (LDBEN) em 1996. Em outros países é conhecido com expressões parecidas. Segundo Stauffer (1), em Portugal, denomina-se Projeto Educativo da Escola; na Argentina, Projeto Educativo Institucional; e na Espanha, Projeto Educativo dos Centros. Esse projeto aponta o caminho a ser seguido por educadores na formação de um profissional, as estratégias a serem adotadas e as metodologias para se atingir o fim, e deve ser estruturado com base nas Diretrizes Curriculares Nacionais.

As DCN/Fisio foram aprovadas, após grandes discussões em que participaram representantes de instituições de ensino superior (IES), a Associação Brasileira de Ensino em Fisioterapia (Abenfisio), o Conselho Federal de Fisioterapia e Terapia Ocupacional (Coffito) e docentes reunidos em fóruns de debates. Após sua aprovação, temos assistido a um avanço no número de cursos de graduação em Fisioterapia, especialmente em instituições particulares. A região Norte apresentou a maior taxa de crescimento desse curso no Brasil entre 1999 e 2004. Até 2002, ano de aprovação das DCN/Fisio, existiam apenas seis cursos nessa região. Atualmente, existem, segundo o cadastro do Instituto Nacional de Estudos e Pesquisas Educacionais Anísio Teixeira (Inep), 27 cursos de fisioterapia nessa região, dos quais apenas quatro $(14,82 \%)$ são mantidos por instituições públicas e $23(85,18 \%)$, por instituições privadas, responsáveis por 4.372 vagas, o que representa um aumento em termos de número de cursos de $350 \%$ e de $233 \%$ em termos de oferta de vagas. 
Aliado a esse aumento no número de cursos, o perfil de graduação definido nas diretrizes mudou o paradigma de formação do profissional fisioterapeuta. Originalmente baseado no aprendizado de técnicas fisioterapêuticas curativas e/ou reabilitativas, buscava a formação de um indivíduo especialista, hoje se busca, por meio do ensino de conteúdos mais amplos, a formação de um indivíduo com competência técnico-científica, generalista e humanista, com capacidade crítica e reflexiva, preparado para atuar no processo saúde-doença pautado em princípios éticos. Segundo a DCN/Fisio (2), os cursos já implantados em 2002 tiveram prazo de dois anos após sua aprovação para se ajustarem à nova diretriz.

A pesquisa tomou como objeto de estudo os projetos pedagógicos dos cursos de Fisioterapia, tendo o objetivo geral de analisar a aderência de tais cursos da região Norte às DCN/Fisio e com os objetivos específicos de: verificar o índice de aderência desses cursos às DCN/Fisio e analisar as potencialidades e fragilidades dos projetos pedagógicos e dos processos concretos de implantação dos cursos em relação às DCN/Fisio.

A aderência é um termo que vem de adhaerentis, particípio presente do verbo latino adhaerere, que significa encontrar-se estreitamente ligado a algo, podendo ser entendido como a aceitação dos princípios de uma ideia, uma doutrina (3). Em nosso estudo consideramos aderência como uma medida de quanto o PPC dos cursos de Fisioterapia se aproxima das DCN/Fisio.

\section{Materiais e métodos}

Para atingir nossos objetivos foi realizado um estudo de caráter analítico-descritivo pautado em uma pesquisa documental. Esse tipo de pesquisa caracteriza-se como a coleta de dados restrita a documentos, contemporâneos ou retrospectivos, escritos ou não, a partir de fontes primárias ou secundárias.

A pesquisa documental é uma das técnicas decisivas para a pesquisa em ciências sociais e humanas. Bardin (4), Minayo (5) e Triviños (6) apontam três etapas para a análise documental: a) pré-análise em que é feita a organização do material, seleção e escolha dos documentos até a leitura exaustiva de todo o conteúdo; b) descrição analítica e exploração do material - quando o material documental é submetido a um estudo aprofundado, havendo a codificação, classificação e categorização dos dados; c) interpretação inferencial, tratamento dos dados e interpretação - que tem início ainda na etapa da pré-análise em que se reexamina o material com o objetivo de aumentar o conhecimento, aprofundar sua visão e estabelecer novas relações. Em nosso estudo, seguimos as etapas sugeridas por esses autores.

As fontes utilizadas foram dez PPC de onze cursos de Fisioterapia, o que representa $37 \%$ do total de cursos (27) existentes na região Norte e cadastrados no Inep. Os PPC foram obtidos por meio dos coordenadores e da home page institucional. A busca das fontes foi realizada, entre os meses de agosto de 2008 e março de 2009. Para fins de resguardar eticamente as instituições, os coordenadores de cursos, os docentes, discentes e egressos, os nomes das instituições foram codificadas em três níveis, sendo o primeiro nível a indicação da categoria administrativa: universidade - UNI, centro universitário - CEU, e faculdade - FAC; o segundo nível, quanto à organização acadêmica, pública (1) e privada (2); e o terceiro nível, uma numeração dada por sorteio (1 a 25).

Na sequência, foi construída a matriz de avaliação, adaptando-se a metodologia adotada pelo grupo de trabalho do Inep que conduziu o estudo de aderência dos cursos de Enfermagem, Medicina e Odontologia às DCN (7). Tal adaptação consistiu na elaboração de uma listagem de indicadores do instrumento de avaliação do Inep que pudessem ser avaliados apenas pela leitura do projeto pedagógico. Para tal, primeiramente foi construída uma primeira listagem de indicadores do instrumento de avaliação (8) que abordasse temas relacionados ao projeto pedagógico, sendo selecionados 32 indicadores do instrumento de avaliação.

Posteriormente, foi construída uma segunda listagem com os indicadores que pudessem ser avaliados apenas pela leitura do projeto pedagógico. Para chegarmos a esses indicadores, utilizamos como critério de exclusão aqueles que de acordo com as instruções do manual do avaliador necessitavam de acesso ao PDI/PPI, os que não se aplicavam aos cursos de Fisioterapia, os que necessitavam de acesso a dados relativos ao corpo docente e técnico-administrativo e os que necessitavam de visita in loco. Assim, dos 32 indicadores chegamos aos 11 usados para avaliação da aderência, listados a seguir: 
Quadro 1 -Indicadores do instrumento de avaliação do Inep que abordam temas relacionados ao PPC e podem ser avaliados apenas pela consulta ao PPC

\begin{tabular}{|c|c|}
\hline 1.4.1 & $\begin{array}{l}\text { Coerência do currículo com os objetivos } \\
\text { do curso }\end{array}$ \\
\hline 1.4 .2 & $\begin{array}{l}\text { Coerência do currículo com o perfil } \\
\text { desejado do egresso }\end{array}$ \\
\hline 1.4 .3 & $\begin{array}{l}\text { Coerência do currículo face às diretrizes } \\
\text { curriculares nacionais }\end{array}$ \\
\hline 1.4 .4 & $\begin{array}{l}\text { Adequação da metodologia de ensino à } \\
\text { concepção do curso }\end{array}$ \\
\hline 1.4 .5 & $\begin{array}{l}\text { Inter-relação das unidades de estudo na } \\
\text { concepção e execução do currículo }\end{array}$ \\
\hline 1.4.6 & Dimensionamento das unidades de estudo \\
\hline 1.4 .7 & $\begin{array}{l}\text { Adequação e atualização das ementas e } \\
\text { programas das unidades de estudo }\end{array}$ \\
\hline 1.4 .8 & Adequação e atualização da bibliografia \\
\hline 1.6 .7 & $\begin{array}{l}\text { Abrangência das atividades e áreas de } \\
\text { formação }\end{array}$ \\
\hline 1.6 .8 & Adequação da carga horária \\
\hline 1.7.1 & $\begin{array}{l}\text { Mecanismos efetivos de acompanhamento } \\
\text { e de cumprimento do trabalho de conclusão } \\
\text { de curso }\end{array}$ \\
\hline
\end{tabular}

Fonte: Dados da pesquisa.

Chamamos a atenção para o fato de que a metodologia utilizada neste estudo é uma adaptação da utilizada pelo Inep em 2006 ao avaliar a aderência dos cursos de Enfermagem, Medicina e Odontologia às respectivas diretrizes curriculares. No estudo conduzido pelo Inep, inicialmente foi elaborada uma listagem dos itens do instrumento de avaliação do Inep para fins de reconhecimento ou renovação de reconhecimento que abordem temas apresentados nas DCN. Posteriormente, de posse dessa listagem, uma comissão composta pelos professores que faziam parte da Comissão Assessora de Avaliação do Inep para esses cursos indicaram, dentre os itens constantes nessa primeira lista, aqueles que estavam fortemente indicados nas DCN. Por um processo de escrutínio, foram selecionados os mais votados. Após esse processo, por meio de métodos estatísticos, escolheram-se os dez indicadores fortemente associados às DCN. Os resultados do estudo comprovaram que essa última listagem mostrou-se mais eficaz para avaliar a aderência dos cursos que as três anteriores.

De posse desses 11 indicadores partimos para a construção de categorias para conduzir uma análise qualitativa global dos PPC. A produção das categorias foi feita para que pudéssemos, a partir da leitura, conhecer os PPC na sua inteireza, a fim de procedermos com mais segurança às etapas seguintes, voltadas especificamente para a obtenção do Índice de Aderências às DCN.

As categorias de análise, segundo Minayo (9), são empregadas para estabelecer classificações, representando um conceito que abrange elementos ou aspectos com características comuns ou que se relacionam entre si. Bardin (4) afirma que, ao classificar os elementos comuns de um conjunto, isso pode ser feito segundo critérios semânticos (temas), sintáticos (verbos, adjetivos) léxicos (sentido da palavra) ou expressivos (classificar as perturbações da linguagem).

Em nosso estudo, a categorização se deu pelo critério semântico, sendo selecionadas oito categorias, a partir de um cruzamento entre os 11 indicadores selecionados na Fase 2 e as Diretrizes Curriculares Nacionais para os Cursos de Graduação em Fisioterapia: 1 - objetivos do curso; 2 - perfil do egresso; 3 - dimensionamento da carga horária, matriz curricular, ementas e programas de disciplinas; 4 - formas de realização da interdisciplinaridade; 5 - modos de integração entre teoria e prática; 6 - Trabalho de Conclusão de Curso; 7 - atividades complementares; 8 - processos de avaliação e metodologias de ensino. Na Fase 4 procedemos à análise propriamente dita dos PPC.

Posteriormente, obtivemos o Índice de Aderência (IA), considerando-se todas as categorias que podem ser assumidas na pontuação, conforme estabelece o instrumento de avaliação do Inep, isto é, (1) muito fraco, (2) fraco, (3) regular, (4) bom e (5) muito bom. Os critérios de pontuação foram aqueles que constam no instrumento de avaliação do Inep.

O IA consiste na razão da diferença entre a soma dos escores obtidos por curso e a soma dos escores mínimos possíveis de serem obtidos com a diferença entre a soma dos escores máximos possíveis e a soma dos escores mínimos possíveis, sendo calculado por meio da seguinte Fórmula 1:

$$
\mathrm{IA}=\frac{\left(\sum \text { escores }\right)-\min \left(\sum \text { escores }\right)}{\max \left(\sum \text { escores }\right)-\min \left(\sum \text { escores }\right)}
$$


Para a identificação das potencialidades e fragilidades, agrupamos os cursos segundo intervalos interquartis a partir do resultado da aplicação do Índice de Aderência. De posse desse agrupamento, realizamos uma análise qualitativa dos PPC dos cursos de Fisioterapia mantidos por IES na região Norte que estivessem nos $1^{\text {o }}$ e $4^{\circ}$ intervalos interquartis, com o propósito de identificar as potencialidades e fragilidades, destacando os pontos fortes e os pontos fracos dos projetos pedagógicos.

\section{Resultados}

A avaliação dos cursos mostrou uma aderência média de $74 \%$, sendo os conceitos obtidos por curso expressos no Quadro 2.

Quadro 2 - Resultado do IA dos dez cursos analisados

\begin{tabular}{lccccc}
\hline IES & UNI122 & UNI208 & UNI212 & UNI104 & CEU124 \\
IA & 0,86 & 0,86 & 0,57 & 0,77 & 0,68 \\
\hline IES & CEU223 & FAC219 & FAC225 & FAC214 & FAC206 \\
IA & 0,95 & 0,64 & 0,70 & 0,75 & 0,68 \\
\hline
\end{tabular}

Fonte: Dados da pesquisa.

Observamos que apenas três cursos (UNI122, UNI208 e CEU223) obtiveram aderência às DCN/ Fisio acima de $80 \%$ no IA1, existindo um curso (UNI212) com menos de $60 \%$ de aderência.

A média do Índice de Aderência (74\%) pode ser considerada abaixo das expectativas. No entanto, observa-se que foram correspondentes as médias encontradas no estudo do Inep realizado em 2006 para os cursos de Enfermagem, cujo valor médio do índice correspondente ao IA foi de 72\% (a média de Medicina foi de 55\%). A tabela com a apresentação da média desse índice para o curso de Odontologia repete a tabela do curso de Enfermagem no trabalho do Inep, não sendo por isso considerada em nosso estudo. Ressalta-se que em nosso trabalho foram dez cursos avaliados enquanto no do Inep foram considerados todos os cursos do Brasil que já haviam passado pelo processo de reconhecimento (45 de Odontologia, 110 de Enfermagem e 33 de Medicina).

Ao analisarmos as médias segundo a categoria administrativa, observamos que os cursos mantidos por instituições públicas obtiveram média dos valores de aderência às DCN/Fisio IA (77\%) maiores do que os cursos mantidos por instituições privadas (73\%), embora, em razão do tamanho da amostra, essa diferença não possa ser considerada estatisticamente significativa (Gráfico 1).

Da mesma forma, ao analisarmos as médias segundo a organização acadêmica, observamos que os centros universitários (81\%) obtiveram média dos valores do Índice de Aderência maiores do que as universidades $(76 \%)$ e do que as faculdades (69\%), embora, pela mesma razão, isso não possa ser considerado estatisticamente significante (Gráfico 2).

A fim de obtermos a classificação dos cursos de acordo com o intervalo interquartil para realizarmos a quarta etapa de nosso estudo, realizamos a análise dos quartis obtendo-se os dados que podem ser observados na Tabela 1.

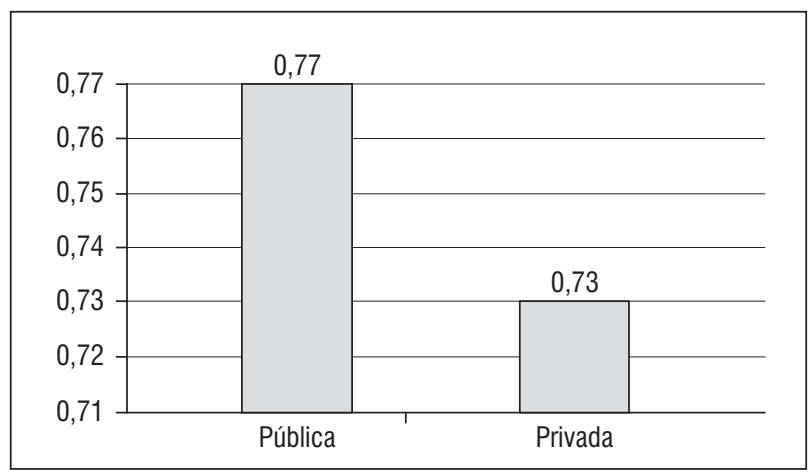

Gráfico 1 - Média dos valores obtidos no Índice de Aderência por categoria administrativa

Fonte: Dados da pesquisa.

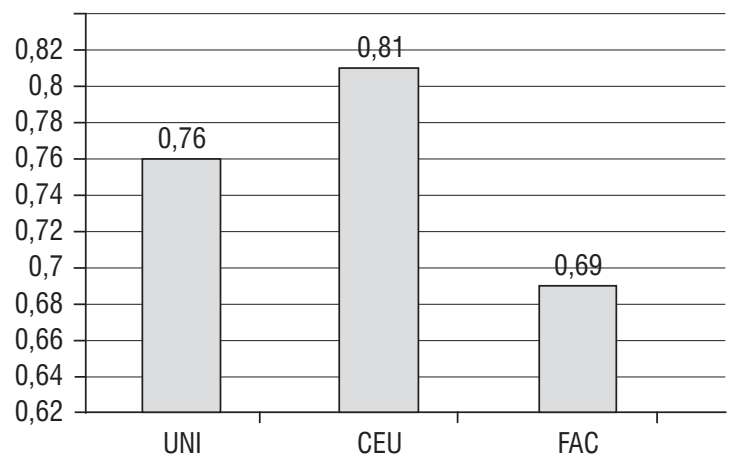

Gráfico 2 - Média dos valores obtidos no Índice de Aderência por organização acadêmica

Fonte: Dados da pesquisa. 
Tabela 1 - Valores dos quartis para os Índices de Aderência

\begin{tabular}{lcc}
\hline & & Índice de Aderência \\
\cline { 3 - 3 } & Válidos (n) & 10 \\
\hline \multirow{3}{*}{ Quartis } & 25 & 0,6700 \\
& 50 & 0,7250 \\
& 75 & 0,8600 \\
\hline
\end{tabular}

Fonte: Dados da pesquisa.

Consideramos, para análise das potencialidades e fragilidades, os cursos com IA menor ou igual a 0,6700 e aqueles com IA maior ou igual a 0,8600 .

De acordo com os resultados, três cursos se encontram no primeiro intervalo interquartil (UNI122, UNI208 e CEU223), e dois cursos encontram-se no quarto intervalo interquartil (UNI212, FAC219).

De acordo com a análise, os três cursos cuja pontuação obtida no Índice de Aderência está no primeiro intervalo interquartil apresentaram as seguintes potencialidades:

1) indicativos de que o projeto pedagógico foi construído coletivamente;

2) matriz curricular com construção interdisciplinar e metodologias de ensino que propiciam a interdisciplinaridade;

3) metodologias de ensino que propiciam a integração teoria-prática;

4) organização e mecanismos de acompanhamento do Trabalho de Conclusão de Curso;

5) abrangência das áreas de formação durante o estágio supervisionado;

6) incentivo à produção científica;

7) dimensionamento da carga horária privilegiando o equilíbrio entre os conteúdos essenciais.

Ainda na análise desses três cursos observamos as seguintes fragilidades:

1) projeto pedagógico sem construção coletiva;

2) unidades de estudo com o dimensionamento privilegiando apenas o aprendizado dos conhecimentos técnicos.

De acordo com a análise, os cursos cuja pontuação obtida no Índice de Aderência está no quarto intervalo interquartil apresentam a seguinte potencialidade:

- presença de regulamento das atividades complementares.

Foram encontradas as seguintes fragilidades nesses cursos:

1) indicativos de que o projeto pedagógico foi elaborado por uma comissão;

2) transcrição literal de elementos das DCN/ Fisio no projeto pedagógico;

3) metodologias de ensino não condizentes com a formação desejada;

4) metodologias de ensino que não privilegiem a interdisciplinaridade e a integração teoria-prática;

5) ausência de elementos que mostrem a organização, o acompanhamento e a orientação efetiva do TCC;

6) ausência de inter-relação entre as unidades de estudo;

7) unidades de estudo privilegiando o aprendizado dos conhecimentos técnicos;

8) pouca abrangência das áreas de formação durante o estágio supervisionado.

\section{Considerações finais}

O projeto pedagógico é o elemento dinâmico normatizador de um curso, construído coletivamente e que deve indicar não apenas o conjunto de disciplinas que devam ser cursadas pelos alunos, mas também as estratégias que devam ser seguidas pelos docentes para atingir os objetivos do curso, devendo para tal ter afinidade com as DCN. As DCN são as normas gerais a serem adotadas pelas IES para cada curso na estruturação de seu projeto pedagógico. Ristoff e Giolo (10) afirmam que o "PPC é, pois a alma do curso, a ponte que une as DCN às práticas pedagógicas propostas pelo currículo".

Ao buscar os indicadores do instrumento de avaliação utilizado pelo Inep para o reconhecimento ou renovação do reconhecimento dos cursos de graduação, modelo abril de 2006, que contivessem temas relativos aos projetos pedagógicos dos cursos, tínhamos o objetivo de analisar o grau de aderência dos PPC às DCN/Fisio. Tão importante quanto encontrar esses 
dados quantitativos foi, a partir dos resultados da aderência, buscar na leitura dos PPC os elementos facilitadores e as fragilidades que interferem positivamente ou negativamente nessa aderência às DCN/Fisio.

De igual importância se revestiram as visitas aos cursos, cujo resultado da aderência às DCN/Fisio se encontrava no primeiro e no quarto intervalos interquartis dentre os dez cursos analisados, em que se buscou o desvendamento das estratégias utilizadas para vencer as fragilidades e confirmar como são trabalhadas as potencialidades.

Este estudo ao analisar as aderências dos PPC às DCN/Fisio, bem como as potencialidades e fragilidades dos cursos com o resultado da aderência nos intervalos interquartis extremos, aponta para a necessidade de que o PPC seja construído coletivamente e, no caso de ter sido elaborado por uma comissão, deva ser do conhecimento de todo corpo docente e discente, da existência de estratégias que promovam a interdisciplinaridade e a integração entre a teoria e a prática, deva existir maior controle sobre os TCC, maior equilíbrio entre a carga horária dos conteúdos essenciais, a diversificação dos cenários de prática, o equilíbrio entre a carga horária desses cenários de prática e que o PPC sofra uma constante avaliação e reajustamento.

$\mathrm{A}$ aderência média dos PPC às DCN/Fisio em $74 \%$ está aquém do desejado, porém está de acordo com a aderência média dos cursos de Enfermagem às suas diretrizes curriculares (77\%), embora esteja bem acima da aderência dos cursos de Medicina às suas diretrizes curriculares (52\%) em estudo realizado pelo Inep (8). A maior aderência encontrada neste estudo, nos PPC de cursos mantidos por universidades e centros universitários em relação àqueles mantidos por faculdades isoladas ou faculdades integradas, também é corroborado pelo estudo do Inep, assim como a maior aderência dos PPC de cursos mantidos por IES públicas em relação às IES privadas. Foi identificada também em nove PPC (dentre os dez analisados) a presença de uma grade curricular constituída de disciplinas isoladas, ainda baseada no modelo de currículo mínimo já extinto pelo MEC, e a existência de ciclos básicos, pré-clínicos e clínicos sem integração entre si, o que dificulta a apropriação do conhecimento pelo aluno.

Apesar dos PPC demonstrarem terem sido elaborados tendo como base as DCN/Fisio, em alguns cursos foi detectada a simples reprodução do discurso das DCN/Fisio sem que, contudo, este seja traduzido em práticas que caracterizem a implantação de tal discurso. Essa reprodução foi destacada pelo estudo do Inep sobre a aderência dos cursos de Enfermagem, Medicina e Odontologia às DCN em PPC dessas três áreas (8).

Também reafirmamos a necessidade dos cursos atenderem ao que estabelece o parágrafo segundo do artigo 32 da Portaria Normativa do Ministério da Educação n. 40, de 12 de dezembro de 2007, sobre a disponibilização dos PPC aos interessados.

Foi considerada, também, a importância da diversidade dos cenários de aprendizagem, fundamental para superar a aprendizagem baseada no conhecimento fisiopatológico e desagregada da realidade sociocultural da população brasileira.

Vale ressaltar que a análise qualitativa buscou aprofundar os achados na análise quantitativa. Assim, índices de aderência às DCN/Fisio apontando, por exemplo, a utilização de ações interdisciplinares por meio de eixos integradores centrais na grade curricular podem ter sido considerados elevados no primeiro momento. No entanto, com a visita ao curso e a entrevista com o coordenador, em um segundo momento mostraram-se fracos, por meio da fala do coordenador ao afirmar que não conseguiam implementar a interdisciplinaridade em razão da não participação dos docentes.

Por outro lado, estratégias de atividades complementares que se mostraram fracas em um primeiro momento, em um determinado curso, por serem baseadas apenas em atividades estritamente técnicas, com a visita ao curso e entrevista com o coordenador, mostraram-se ricas na análise qualitativa por oportunizar ao aluno a prática de atividades que o enriquece na formação como cidadão. Tais esforços, desenvolvidos por grupos que tentam inovar dentro das reais condições de trabalho oferecidas institucionalmente, devem ser destacados e elogiados.

Traçando-se um perfil comparativo entre as características encontradas nas instituições que se encontram no primeiro e no quarto intervalos interquartis, observamos que as do primeiro intervalo tiveram seu PPC construído coletivamente, possuem uma matriz curricular com construção interdisciplinar, usam metodologias de ensino que propiciam a interdisciplinaridade e a integração entre teoria e prática, desenvolvem atividades práticas precocemente, tendo equilíbrio entre os conteúdos essenciais, têm mecanismos que possibilitam o acompanhamento 
do aluno na construção do TCC, têm abrangência nas áreas de formação durante o estágio supervisionado e incentivam a produção científica.

Em oposição, as instituições localizadas no quarto intervalo possuem indicativos de que tiveram seus projetos pedagógicos construídos por uma comissão, possuem transcrição literal de alguns elementos das DCN/Fisio em seus PPC, não propiciam a interdisciplinaridade nem a integração teoria-prática, têm a matriz curricular sem inter-relação entre as unidades de estudo, privilegiam o aprendizado de conhecimento técnico nos ciclos teóricos e as áreas de formação curativa e reabilitativa durante o estágio supervisionado e não mostram no PPC os mecanismos de organização e acompanhamento do TCC.

Ressaltamos a necessidade de que entidades como a Abenfisio, como associação que tem por objetivo subsidiar o desenvolvimento do ensino em Fisioterapia no país, promovam um estudo semelhante com maior abrangência, por meio do acesso ao relatório de avaliação dos todos os cursos de Fisioterapia do Brasil já avaliados pelo Inep.

Para concluir, faz-se necessário explicitar que este estudo não pretendeu ser um estudo acabado, nem indicar os caminhos a serem percorridos pelas instituições para alcançarem as transformações necessárias. Trata-se apenas da apresentação de parte de uma realidade, que evidencia a aderência ou não dos PPC dos cursos às DCN/Fisio.

\section{Referências}

1. Stauffer AB. Projeto político-pedagógico: instrumento consensual e/ou contra-hegemônico à lógica do capital? [tese]. Rio de Janeiro: Pontifícia Universidade Católica do Rio de Janeiro; 2007.

2. Brasil. Conselho Nacional de Educação. Câmara de Educação Superior Resolução n. 4 de 19 de fevereiro de 2002. Institui as Diretrizes Curriculares Nacionais do curso de graduação em Fisioterapia. 2002. [acesso 10 ago. 2010]. Disponível em: http://portal.mec. gov.br/cne/arquivos/pdf/CES 042002.pdf.
3. Houaiss A. Dicionário eletrônico Houaiss da língua portuguesa. São Paulo: Instituto Antonio Houaiss. 2002. CDROM

4. Bardin L. Análise de conteúdo. 3a ed. Portugal: Edições 70; 2004.

5. Minayo MCS. O desafio do conhecimento: pesquisa qualitativa em saúde. 8a ed. São Paulo; Rio de Janeiro: Hucitec; Abrasco; 2004.

6. Triviños ANS. Introdução à pesquisa em ciências sociais: a pesquisa qualitativa em educação. São Paulo: Atlas; 1987.

7. Brasil. Avaliação dos cursos de graduação: instrumento. Brasília: Instituto Nacional de Estudos e Pesquisas Educacionais Anísio Teixeira; 2006.

8. Brasil. Ministério da Educação e Ministério da Saúde. A aderência dos cursos de graduação em Enfermagem, Medicina e Odontologia às Diretrizes Curriculares Nacionais. Brasília: Ministério da Saúde; 2006.

9. Minayo MC. Pesquisa social: teoria, método e criatividade. 20a ed. Petrópolis: Vozes; 2002.

10. Ristoff D, Giolo, J. O Sinaes como sistema. Rev Bras de Pós-Graduação. 2006;3(6):193-213.

Recebido: 24/11/2010

Received: 11/24/2010

Aprovado: 26/05/2011

Approved: 05/26/2011 